\title{
Alveolar Rhabdomyosarcoma of the Paranasal Cavity in an Adult Woman
}

\author{
Renato Covello ${ }^{a}$, Stefano Licci ${ }^{\mathrm{b}, \mathrm{f}}$, Barbara Pichic, Virginia Ferraresi ${ }^{\mathrm{d}}$, \\ Antonello Vidirie, Giuseppe Spriano ${ }^{\mathrm{c}}$
}

\begin{abstract}
Alveolar rhabdomyosarcoma (RMS) is extremely rare in adults older than 45 years and may be very difficult to distinguish from other primitive round cell neoplasms without immunohistochemistry and/or genetic studies. In adults, the differential diagnosis of small round cell tumors of the head and neck region includes small cell carcinoma, lymphomas and neuroepithelial tumors, such as esthesioneuroblastoma and, as last choice, rhabdomyosarcoma. Initial immunoprofiling may not include myogenic markers. Moreover, aberrant expression of epithelial and neuroendocrine markers in alveolar RMS may be a potentially serious diagnostic pitfall. In this report, we describe the clinico-pathological features of a case of paranasal alveolar RMS in a 61-year-old woman with no previous history of neoplasia and discuss the differential diagnosis of small round cell tumor in the nasal/paranasal cavity.
\end{abstract}

Keywords: Alveolar rhabdomyosarcoma; Adult; Nasal cavity; CD56; Myogenin

\section{Introduction}

Rhabdomyosarcomas are a morphologically and clinically

\footnotetext{
Manuscript accepted for publication August 23, 2013

aDepartment of Pathology, National Cancer Institute "Regina Elena", Rome, Italy

bepartment of Pathology, "Santo Spirito" Hospital, Rome, Italy

${ }^{\mathrm{c}}$ Department of Otorhinolaryngology Head and Neck Surgery, National Cancer Institute "Regina Elena", Rome, Italy

dDepartment of Oncology, National Cancer Institute "Regina Elena", Rome, Italy

e Department of Diagnostic Imaging, National Cancer Institute "Regina Elena", Rome, Italy

${ }^{\mathrm{f}}$ Corresponding author: Stefano Licci, Department of Pathology, Santo Spirito Hospital, Lungotevere in Sassia, 1, 00193 Rome, Italy.

Email: stefano.licci@hotmail.it
}

doi: http://dx.doi.org/10.4021/jmc1498e heterogeneous family of malignant soft tissue tumors related to the myogenic lineage [1, 2]. Alveolar rhabdomyosarcoma (RMS) and embryonal RMS represent the two main histological patterns and must be differentiated from other small round cell tumors. RMS is the most common soft tissue sarcoma in the pediatric population, comprising approximately $5 \%$ of all childhood tumors and nearly $50 \%$ of soft tissue sarcomas arising in 0 - 14 years old children [3-5]. In contrast, it is remarkably uncommon in elder adults, representing merely $2-5 \%$ of all malignant soft tissue tumors, mostly of the pleomorphic type [6].

Alveolar RMS may involve the head and neck region $(40 \%)$, the genitourinary system $(20 \%)$, the extremities (20\%), the trunk (10\%) and other sites (10\%) [7-10].

Here we report an uncommon case of alveolar RMS in the paranasal cavity of a 61-year-old woman, with emphasis on the differential diagnosis of small round cell tumors in this location and the potential immunohistological diagnostic pitfalls.

\section{Case Report}

A 61-year-old female presented for onset of otalgia and a lump in the right neck.

A computed tomography (CT) scan revealed a right ethmoidal sinus lesion extending to the homolateral maxillary sinus and medial turbinate, with radiological features suspicious for a neoplasia (Fig. 1A, B).

A biopsy was performed and histological evaluation revealed an infiltrative neoplastic proliferation composed of sheets and solid nests of atypical small round cells (Fig. 2A), displaying hypercromatic nuclei, scant eosinophilic cytoplasm and brisk mitotic activity (Fig. 2B). Examination of the individual cell morphology was often limited by variable crush artifacts and necrosis. On the basis of the sole morphology, an initial diagnosis of malignant undifferentiated neoplasia was made, being a small cell carcinoma the favored diagnostic hypothesis. Immunohistochemistry was performed using an antibody panel (Table 1) that could confirm the hypothesis of small cell undifferentiated carcinoma (EMA, cytokeratins AE1/AE3, CK7, CK20), including neu- 


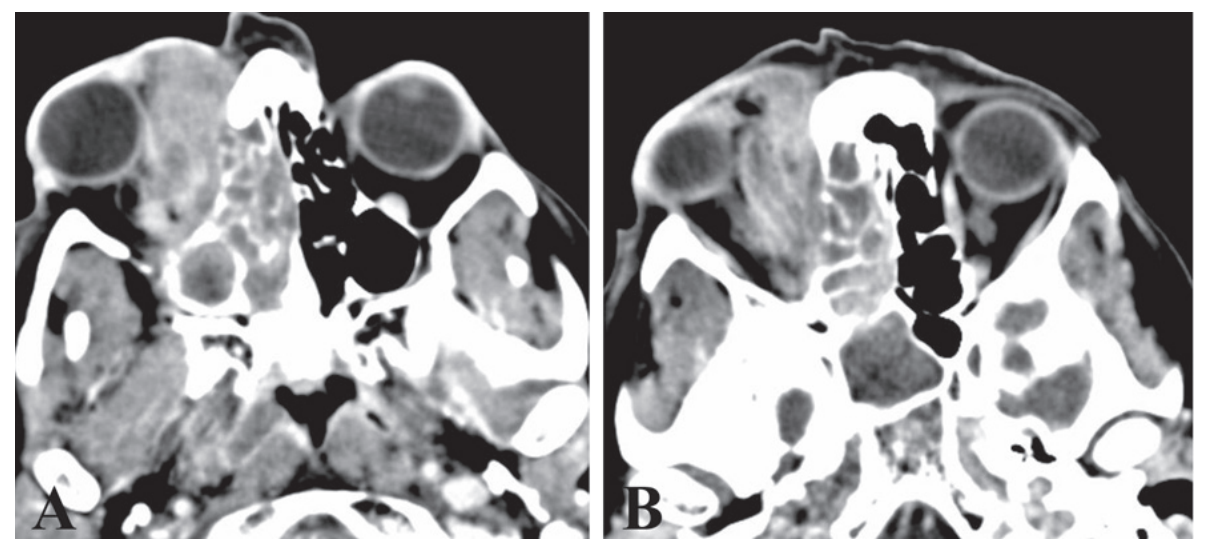

Figure 1. CT scan reveals a right ethmoidal sinus lesion (A), $30 \mathrm{~mm}$ in diameter, extending to the homolateral maxillary sinus and medial turbinate, and infiltrating the right orbital cavity (B).

roendocrine markers (CD56, chromogranin, synaptophysin) and GFAP, s-100 protein, HMB45, melan-A, CD45, EBVLMP1 and myeloperoxidase (for excluding neuroectodermal tumors, melanoma and lymphohemathopoietic neoplasms).

The tumor cells showed a positive immunostain only for CD56 (Fig. 2C). A sarcomatous nature of the lesion was suspected and appropriate additional immunohistochemical studies were performed. The neoplastic cells displayed a positive immunoreaction for vimentin, desmin (Fig. 2D), MyoD1 and myogenin (Fig. 2E), suggesting a rhabdomyosarcomatous differentiation.

A cytogenetic analysis revealed the $\mathrm{t}(2 ; 13)$ translocation, a chromosomal rearrangement typical of alveolar RMS.

On the basis of immunohistological and cytogenetic findings a final diagnosis of alveolar RMS was made.

In January 2010, the patient underwent the first cycle of polichemotherapy treatment (VAC scheme: vincristine $2 \mathrm{mg}$ / day 1 , adriamicine $108 \mathrm{mg}$ /day 1, cyclophosfamide 3,024/ days 1-2).

One month later the patient received the second cycle ( $75 \%$ of VAC scheme: vincristine $1.5 \mathrm{mg} /$ day 1 , adriamicine $80 \mathrm{mg} /$ day 1 , cyclophosfamide 2,268/days 1-2). The third cycle was at $66 \%$ of VAC scheme for progressive mielotoxicity side effect.

CT scan and magnetic resonance imaging (MRI) performed in April revealed a good local response with $80 \%$ of tumor regression. From May to July the patient was treated with external beam radiation therapy ( $69.3 \mathrm{~Gy} / 33$ fraction). One month later MRI revealed the presence of local residual disease.

The case was discussed by the Head and Neck Tumor Management Team that scheduled the patient for surgery. Before the operation, the patient presented with a new onset of pain at the right arm and scapula. PET-CT scan and bone scintigraphy revealed the presence of multiple bone metastases. The patient was referred to palliative chemotherapy and unfortunately died for disease complications one year after the initial consultation.

\section{Discussion}

Alveolar RMS is composed in its classical form of distinctive nests of primitive-appearing round cells, which grows in a discohesive fashion, surrounded by hyalinized and highly vascular fibrous septa producing a pattern reminiscent of the alveoli of the lung. Obvious rhabdomyoblastic differentiation, in the form of strap cells and cytoplasmic cross striations, is rarely identified.

Solid forms of alveolar RMS lack the prominent nested pattern and cellular discohesion seen in the classical histotype and may closely mimic a variety of other small round cell tumors, such as small cell carcinoma, lymphoma, neuroblastoma and malignant melanoma [11].

In immunohistochemistry, alveolar RMS typically expresses vimentin and muscle-specific antigens, such as desmin, muscle actins (including smooth muscle isoforms), myogenin and MyoD1 $[12,13]$. Aberrant expression of epithelial and neuroendocrine markers, a potentially serious diagnostic pitfall, has been recently described $[14,15]$.

Alveolar RMS is a very aggressive tumor with a poor prognosis and requires a histotype-specific aggressive therapy. Therefore an accurate diagnosis and its distinction from other small round cell tumors are of crucial clinical importance.

While some cases of alveolar RMS have been described in young adults, the occurrence of this neoplasm in adults over the age of 45 years is extremely uncommon, being pleomorphic RMS, characterized by different microscopic features and biological behavior, the most common histotype in this category of patients $[8-10,16]$. For this reason, the inclusion of alveolar RMS in the differential diagnosis of 
Table 1. Details on Antibodies Used for the Immunohistochemical Study

\begin{tabular}{|c|c|c|c|c|}
\hline Antibody & Clone & Source & Dilution & Pretreatment \\
\hline GFAP & $6 \mathrm{~F} 2$ & Dako & $1: 50$ & Microwave \\
\hline Chromogranin A & DAK-A3 & Dako & $1: 100$ & Microwave \\
\hline Vimentin & 3B4 & Ventana & No & Pressure cooker \\
\hline Desmin & D33 & Dako & $1: 50$ & Microwave \\
\hline CD56 & $123 \mathrm{C} 3$ & Dako & No & Microwave \\
\hline CK20 & K20.8 & Dako & No & Microwave \\
\hline CK7 & OV-TL 12/30 & Dako & $1: 50$ & Microwave \\
\hline Cytokeratin & AE1/AE3 & Dako & $1: 50$ & Pressure cooker \\
\hline S-100 protein & Polyclonal & Dako & $1: 400$ & Microwave \\
\hline HMB45 & HMB45 & Novocastra & $1: 30$ & Microwave \\
\hline MART1 & A103 & Dako & $1: 25$ & Microwave \\
\hline Synaptophysin & SY38 & Dako & No & Microwave \\
\hline EMA & GP1.4 & Novocastra & No & Microwave \\
\hline $\mathrm{CD} 45$ & $2 \mathrm{~B} 11+\mathrm{PD} 7 / 26$ & Dako & No & Microwave \\
\hline Myogenin & F5D & Dako & No & Microwave \\
\hline EBV-LMP1 & CS.1-4 & Dako & No diluition & Microwave \\
\hline Myeloperoxidase & Polyclonal & Dako & $1: 300$ & Microwave \\
\hline MYOD1 & $5.8 \mathrm{~A}$ & Dako & $1: 50$ & Pressure cooker \\
\hline
\end{tabular}

small round cell tumors of the head and neck region is often neglected. Other malignancies such as small cell undifferentiated or neuroendocrine carcinoma, lymphoma, olfactory neuroblastoma and melanoma are more likely to be considered in this location in elder patients.

In our case, the initial diagnostic work-up for an undifferentiated tumor arising in the head and neck region in a 56-year-old female patient did not include myogenic markers. The neoplastic cells were positive for CD56 immunostain and only as a consequence of no immunoreaction for the other epithelial, lymphoma, or malignant melanoma markers, the differential diagnosis was expanded to include RMS and additional immunostains for a myogenic differentiation were pursued.

The differential diagnosis of CD56 positive small round cell tumors includes natural killer (NK)-cell tumor, rhabdomyosarcoma, small cell carcinoma, neuroblastoma, primary neuroectodermal tumor, malignant peripheral nerve sheath tumor, desmoplastic small round cell tumor, synovial sarcoma and malignant melanoma.

CD56 expression has an established role in the diagnosis of non-Hodgkin lymphoma/NK cell type and other hematological malignancies. The sinonasal region is notably a common location for $\mathrm{NK} / \mathrm{T}$-cell lymphomas, in particular in some areas of the world [17].

CD56 immunostain is of limited value in the differential diagnosis of sarcomas because it is widely expressed in these neoplasms. However, it may be useful in combination with the assessment of CD99 expression in the differential diagnosis with Ewing's sarcoma. Only 10-25\% of Ewing's sarcomas are CD56 positive in contrast to $100 \%$ embryonal and alveolar RMSs [18, 19].

CD56 is also commonly expressed in neuroendocrine tumors and the co-expression of synaptophysin and/or chromogranin is highly suggestive of neuronal or neuroendocrine differentiation. 


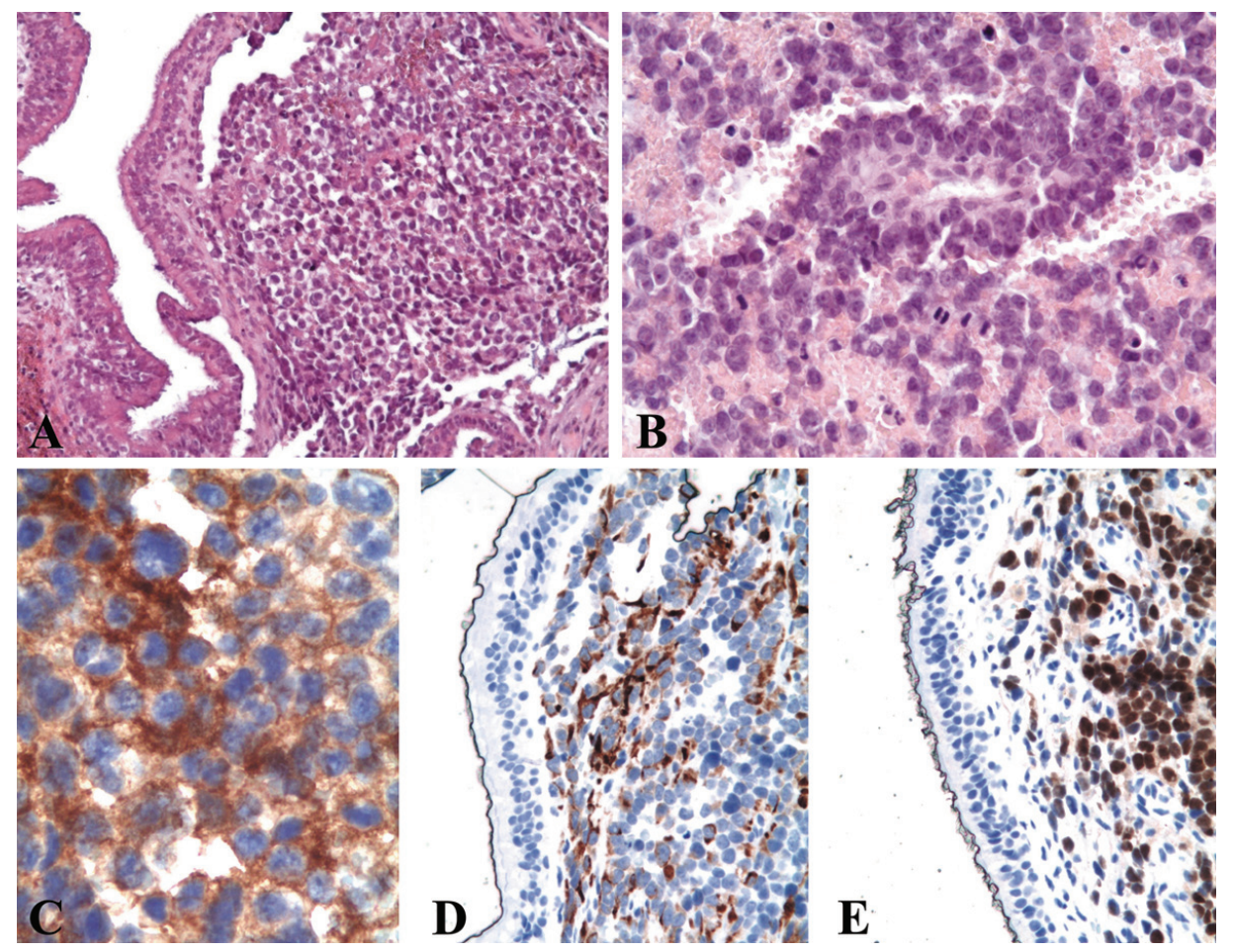

Figure 2. Histological examination of the biopsy shows a submucosal infiltrative lesion composed of sheets of small cells $(A$, hematoxylin and eosin, original magnification $\times 200$ ) displaying round hyperchromatic nuclei with nucleoli, scant eosinophilic cytoplasm and brisk mitotic activity (B, hematoxylin and eosin, original magnification $\times 400)$. At immunohistochemistry, the neoplastic cells show a diffuse cytoplasmic positivity for CD56 (C, original magnification $\times 630)$ and desmin (D, original magnification $\times 400)$ and diffuse nuclear positivity for myogenin $(E$, original magnification $\times 400)$.

Indeed, some cases of synaptophysin positive immunoreaction in alveolar RMS have been reported $[14,15]$. Conversely, rhabdomyoblastic differentiation may occur in olfactory neuroblastoma [20].

The differential diagnosis of poorly differentiated round cell neoplasms represents a common problem. In most cases morphologic evaluation is not sufficient and further investigations are required. The study of the immunohistochemical profile of the tumor is mandatory and the evaluation of the right combination of immuno-markers may have very important prognostic and therapeutic implications. In doubtful cases, cytogenetics and molecular biology studies can be useful in establishing the right diagnosis or to confirm the diagnostic hypothesis. The most common chromosomal rearrangements in alveolar RMS are $\mathrm{t}(2 ; 13)(\mathrm{q} 35 ; \mathrm{q} 14)$, which is seen in $55 \%$ of cases, and $\mathrm{t}(1 ; 13)(\mathrm{p} 3 ; \mathrm{q} 14)$, which is encountered in about $22 \%$ of cases [11].

Our case suggests that in cases of undifferentiated round cell neoplasms of the head and neck region in elder patients, alveolar RMS should be taken into consideration in the differential diagnosis, along with lymphoma, small cell carcinoma and estesioneuroblastoma that are commonly encountered in such clinical spectrum. Therefore, the immunohistochemical differential diagnosis should always include a myogenic marker, such as desmin, myogenin or MyoD1. The performance of one more immunostain can avoid a diagnostic pitfall and provide a more rapid and correct diagnosis. Moreover, the demonstration of specific chromosomal rearrangements may be a very helpful diagnostic tool in doubtful cases.

\section{References}

1. Weiss SW, Goldblum JR. Enzinger and Weiss's Soft Tissue Tumors, 4th ed. Mosby: St. Louis, 2001.

2. Fletcher CDM, Unni KK, Mertens F. (Eds). World Health Organization Classification of tumours. Pathology and genetics of tumours of soft tissue and bone. IARC Press: Lyon, 2002.

3. Crist W, Gehan EA, Ragab AH, Dickman PS, Donaldson SS, Fryer C, Hammond D, et al. The Third Intergroup Rhabdomyosarcoma Study. J Clin Oncol. 1995;13(3):610-630.

4. Enzinger FM, Shiraki M. Alveolar rhabdomyosarcoma. An analysis of 110 cases. Cancer. 1969;24(1):18-31.

5. Caillaud JM, Gerard-Marchant R, Marsden HB, van Unnik AJ, Rodary C, Rey A, Flamant F. Histopathological 
classification of childhood rhabdomyosarcoma: a report from the International Society of Pediatric Oncology pathology panel. Med Pediatr Oncol. 1989;17(5):391-400.

6. Hawkins WG, Hoos A, Antonescu CR, Urist MJ, Leung DH, Gold JS, Woodruff JM, et al. Clinicopathologic analysis of patients with adult rhabdomyosarcoma. Cancer. 2001;91(4):794-803.

7. Tsokos M, Webber BL, Parham DM, Wesley RA, Miser A, Miser JS, Etcubanas E, et al. Rhabdomyosarcoma. A new classification scheme related to prognosis. Arch Pathol Lab Med. 1992;116(8):847-855.

8. Callender TA, Weber RS, Janjan N, Benjamin R, Zaher M, Wolf P, el-Naggar A. Rhabdomyosarcoma of the nose and paranasal sinuses in adults and children. Otolaryngol Head Neck Surg. 1995;112(2):252-257.

9. Ahmed AA, Tsokos M. Sinonasal rhabdomyosarcoma in children and young adults. Int J Surg Pathol. 2007;15(2):160-165.

10. Lanzkowsky P. Rhabdomyosarcoma and other soft tissue sarcomas. In: Manual of Pediatric Hematology and Oncology, vol 1. 3rd ed. New York: Academic Press, 2000, chapter 20 .

11. Parham DM. Pathologic classification of rhabdomyosarcomas and correlations with molecular studies. Mod Pathol. 2001;14(5):506-514.

12. Cessna MH, Zhou H, Perkins SL, Tripp SR, Layfield L, Daines C, Coffin CM. Are myogenin and myoD1 expression specific for rhabdomyosarcoma? A study of 150 cases, with emphasis on spindle cell mimics. Am J Surg Pathol. 2001;25(9):1150-1157.
13. Seidal T, Kindblom LG, Angervall L. Myoglobin, desmin and vimentin in ultrastructurally proven rhabdomyomas and rhabdomyosarcomas. An immunohistochemical study utilizing a series of monoclonal and polyclonal antibodies. Appl Pathol. 1987;5(4):201-219.

14. Bahrami A, Gown AM, Baird GS, Hicks MJ, Folpe AL. Aberrant expression of epithelial and neuroendocrine markers in alveolar rhabdomyosarcoma: a potentially serious diagnostic pitfall. Mod Pathol. 2008;21(7):795806.

15. Leroy X, Petit ML, Fayoux P, Aubert S, Escande F. Aberrant diffuse expression of synaptophysin in a sinonasal alveolar rhabdomyosarcoma. Pathology. 2007;39(2):275-276.

16. Moon HS, Kwon SW, Lee JH. A case of alveolar rhabdomyosarcoma of the ethmoid sinus invading the orbit in an adult. Korean J Ophthalmol. 2006;20(1):70-75.

17. Gaal K, Sun NC, Hernandez AM, Arber DA. Sinonasal NK/T-cell lymphomas in the United States. Am J Surg Pathol. 2000;24(11):1511-1517.

18. Garin-Chesa P, Fellinger EJ, Huvos AG, Beresford HR, Melamed MR, Triche TJ, Rettig WJ. Immunohistochemical analysis of neural cell adhesion molecules. Differential expression in small round cell tumors of childhood and adolescence. Am J Pathol. 1991;139(2):275-286.

19. Miettinen M, Cupo W. Neural cell adhesion molecule distribution in soft tissue tumors. Hum Pathol. 1993;24(1):62-66.

20. Slootweg PJ, Lubsen H. Rhabdomyoblasts in olfactory neuroblastoma. Histopathology. 1991;19(2):182-184. 